\title{
A NOTE ON SOLUTIONS OF INTERVAL-VALUED VOLTERRA INTEGRAL EQUATIONS
}

\author{
TRUONG VINH AN, NGUYEN DINH PHU AND NGO VAN HOA \\ Communicated by Paul Martin
}

\begin{abstract}
In this paper we consider the interval-valued Volterra integral equations (IVIEs). We study the problem of existence and uniqueness of solutions for IVIEs. Finally, we give some examples for IVIEs.
\end{abstract}

1. Introduction. Set-valued differential and integral equations are an important part of the theory of set-valued analysis, and they play an important role in the theory and application of control theory. They were first studied in 1969 by De Blasi and Iervolino [4]. Recently, set-valued differential equations have been studied by many scientists due to their applications in many areas. For the basic theory on setvalued differential and integral equations, readers can be referred to the following books and papers (see $[\mathbf{1}, \mathbf{2}, \mathbf{5 - 8}, \mathbf{1 1 - 1 5}, \mathbf{1 8}]$ and the references therein).

Interval-valued analysis and interval differential equations (IDEs) are special cases of the set-valued analysis and set-valued differential equations, respectively. In many situations, when modeling real world phenomena, information about the behavior of a dynamic system is uncertain and one has to consider these uncertainties to gain better meaning with full models. Use of the interval-valued differential equation is a natural way to model dynamic systems subject to uncertainties. Recently, much work has been done by several authors in the theory of interval-valued differential equations (see, e.g., $[\mathbf{3}, \mathbf{9}, \mathbf{1 0}, \mathbf{1 7}, \mathbf{1 9}]$ ).

Existence theorems for Volterra integral equations have been studied extensively in view of their applications to predator-prey models and

\footnotetext{
Keywords and phrases. Volterra integral equations, interval-valued integral equations.

This research was funded by the Vietnam National Foundation for Science and Technology Development (NAFOSTED).

The third author is the corresponding author.

Received by the editors on April 13, 2012, and in revised form on April 29, 2013.

DOI:10.1216/JIE-2014-26-1-1 Copyright (C2014 Rocky Mountain Mathematics Consortium
} 
medical diagnosis. In this paper, we generalize such existence theorems to interval-valued mappings using the concept of interval-valued functions and the integral due to Stefanini and Bede [18]. Similarly, as in classical analysis, we would like to introduce four main types of interval-valued integral equations: their names appear in the list below. Suppose that $F:\left[t_{0}, T\right] \rightarrow \mathbf{I}, K:\left[t_{0}, T\right] \times\left[t_{0}, T\right] \rightarrow \mathbf{I}$ are continuous interval-valued functions, $k:\left[t_{0}, T\right] \rightarrow \mathbf{R}$ is a continuous real-valued function and $\lambda$ is a constant. We classify the interval-valued integral equations as follows:

(i) Interval-valued non-homogeneous Volterra integral equation

$$
X(t)=F(t)+\int_{t_{0}}^{t} k(t, s) X(s) d s
$$

(ii) Interval-valued homogeneous Volterra integral equation

$$
X(t)=\int_{t_{0}}^{t} k(t, s) X(s) d s
$$

(iii) Interval-valued non-homogeneous Fredholm integral equation

$$
X(t)=F(t)+\lambda \int_{t_{0}}^{T} k(t, s) X(s) d s
$$

(iv) Interval-valued homogeneous Fredholm integral equation

$$
X(t)=\lambda \int_{t_{0}}^{T} k(t, s) X(s) d s,
$$

where $t \in\left[t_{0}, T\right]$. We have noted that the interval-valued Volterra equation can be considered as a special case of the interval-valued Fredholm equation when $k(t, s)=0$ for $s>t$ in $\left[t_{0}, T\right]$. The function $k(t, s)$ appearing in the above four equations is called the kernel of the interval-valued integral equation. Such a kernel is symmetric if $k(t, s)=k(s, t)$, for all $t, s \in\left[t_{0}, T\right]$.

In this paper, we shall prove the existence and uniqueness theorem of solutions for interval-valued Volterra integral equations of the following form

$$
X(t)=F(t)+\int_{t_{0}}^{t} K(t, s, X(s)) d s
$$


This paper is organized as follows. In Section 2, we recall some basic concepts and notations about interval-valued analysis and intervalvalued differential equations. In Section 3, we present the existence and uniqueness theorem of a solution to the interval-valued Volterra integral equations. Finally, we give some examples for IVIEs.

2. Preliminaries and notation. Let $\mathcal{K}_{c}^{n}$ be the space of non empty compact and convex sets of $\mathbf{R}^{n}$. The set of real intervals will be denoted by $\mathbf{I}$ where $\mathbf{I}=\mathcal{K}_{c}^{1}$. The set interval $[\varnothing, \varnothing]$ is a singleton which contains a single element: $\varnothing \in[\varnothing, \varnothing] . \varnothing=\{0\}=[0,0]$. The addition and scalar multiplication in $\mathbf{I}$ is defined as usual, i.e., for $A, B \in \mathbf{I}, A=\left[a^{-}, a^{+}\right]$, $B=\left[b^{-}, b^{+}\right]$, where $a^{-} \leq a^{+}, b^{-} \leq b^{+}$, and $\lambda \geq 0$, then we have

$A+B=\left[a^{-}+b^{-}, a^{+}+b^{+}\right], \quad \lambda A=\left[\lambda a^{-}, \lambda a^{+}\right], \quad\left(-\lambda A=\left[\lambda a^{+}, \lambda a^{-}\right]\right)$.

Furthermore, let $A \in \mathbf{I}, \lambda_{1}, \lambda_{2}, \lambda_{3}, \lambda_{4} \in \mathbf{R}$ and $\lambda_{3} \lambda_{4} \geq 0$. Then we have $\lambda_{1}\left(\lambda_{2} A\right)=\left(\lambda_{1} \lambda_{2}\right) A$ and $\left(\lambda_{3}+\lambda_{4}\right) A=\lambda_{3} A+\lambda_{4} A$. Let $A, B \in \mathbf{I}$ as above; then the Hausdorff metric $H$ in $\mathbf{I}$ is defined as follows:

$$
H(A, B)=\max \left\{\left|a^{-}-b^{-}\right|,\left|a^{+}-b^{+}\right|\right\} .
$$

We notice that $(\mathbf{I}, H)$ is a complete, separable and locally compact metric space.

We define the magnitude and the length of $A \in \mathbf{I}$ by:

$$
H(A,\{0\})=\|A\|=\max \left\{\left|a^{-}\right|,\left|a^{+}\right|\right\}, \quad \operatorname{len}(A)=a^{+}-a^{-},
$$

respectively.

The Hausdorff metric (2.1) satisfies some of the properties below:

$$
\begin{aligned}
& H(A+C, B+C)=H(A, B) \quad \text { and } \quad H(A, B)=H(B, A), \\
& H(A+B, C+D) \leq H(A, C)+H(B, D), \\
& H(\lambda A, \lambda B)=|\lambda| H(A, B), \\
& H(A, B) \leq H(A, C)+H(C, B),
\end{aligned}
$$

for all $A, B, C, D \in \mathbf{I}$ and $\lambda \in \mathbf{R}$. Let $A, B \in \mathbf{I}$. If there exists an interval $C \in \mathbf{I}$ such that $A=B+C$, then we call $C$ the Hukuhara difference of $A$ and $B$. The interval $C$ is denoted by $A \ominus B$. Note 
that $A \ominus B \neq A+(-) B$. It is known that $A \ominus B$ exists in the case len $(A) \geq$ len $(B)$. Besides that, we can see the following properties for $A, B, C, D \in \mathbf{I}$ (see $[\mathbf{9}, \mathbf{1 0}])$ :

- if $A \ominus B, A \ominus C$ exist, then $H(A \ominus B, A \ominus C)=H(B, C)$;

- if $A \ominus B, C \ominus D$ exist, then $H(A \ominus B, C \ominus D)=H(A+D, B+C)$;

- if $A \ominus B, A \ominus(B+C)$ exist, then there exist $(A \ominus B) \ominus C$ and $(A \ominus B) \ominus C=A \ominus(B+C)$;

- if $A \ominus B, A \ominus C, C \ominus B$ exist, then there exist $(A \ominus B) \ominus(A \ominus C)$ and $(A \ominus B) \ominus(A \ominus C)=C \ominus B$.

Definition 2.1 $[9]$. We say that the interval-valued mapping $F$ : $\left[t_{0}, T\right] \rightarrow \mathbf{I}$ is continuous at the point $t \in\left[t_{0}, T\right]$ if, for every $\varepsilon>0$, there exists $\delta=\delta(t, \varepsilon)>0$ such that, for all $s \in\left[t_{0}, T\right]$ such that $|t-s|<\delta$, one has $H(F(t), F(s)) \leq \varepsilon$.

The strongly generalized differentiability was introduced in $[\mathbf{1 8}]$ and studied in $[\mathbf{3}, \mathbf{9 - 1 5}]$.

Definition $2.2[\mathbf{1 8}]$. Let $X:\left[t_{0}, T\right] \rightarrow \mathbf{I}$ and $t \in\left[t_{0}, T\right]$. We say that $X$ is strongly generalized differentiable of the first-order differential at $t$, if there exists $X^{\prime}(t) \in \mathbf{I}$, such that

(i) for all $h>0$ sufficiently small, there exist $X(t+h) \ominus X(t)$, $X(t) \ominus X(t-h)$ and

$$
\begin{aligned}
& \lim _{h \searrow 0} H\left(\frac{X(t+h) \ominus X(t)}{h}, X^{\prime}(t)\right)=0, \\
& \lim _{h \searrow 0} H\left(\frac{X(t) \ominus X(t-h)}{h}, X^{\prime}(t)\right)=0
\end{aligned}
$$

or

(ii) for all $h>0$ sufficiently small, there exist $X(t) \ominus X(t+h)$, $X(t-h) \ominus X(t)$ and

$$
\begin{aligned}
& \lim _{h \searrow 0} H\left(\frac{X(t) \ominus X(t+h)}{-h}, X^{\prime}(t)\right)=0, \\
& \lim _{h \searrow 0} H\left(\frac{X(t-h) \ominus X(t)}{-h}, X^{\prime}(t)\right)=0
\end{aligned}
$$


or

(iii) for all $h>0$ sufficiently small, there exist $X(t+h) \ominus X(t)$, $X(t-h) \ominus X(t)$ and

$$
\begin{aligned}
& \lim _{h \searrow 0} H\left(\frac{X(t+h) \ominus X(t)}{h}, X^{\prime}(t)\right)=0, \\
& \lim _{h \searrow 0} H\left(\frac{X(t-h) \ominus X(t)}{-h}, X^{\prime}(t)\right)=0
\end{aligned}
$$

or

(iv) for all $h>0$ sufficiently small, there exist $X(t) \ominus X(t+h)$, $X(t) \ominus X(t-h)$ and the limits

$$
\begin{aligned}
& \lim _{h \searrow 0} H\left(\frac{X(t) \ominus X(t+h)}{-h}, X^{\prime}(t)\right)=0, \\
& \lim _{h \searrow 0} H\left(\frac{X(t) \ominus X(t-h)}{h}, X^{\prime}(t)\right)=0 .
\end{aligned}
$$

( $h$ in the denominators means $1 / h)$. In this definition, case (i) ((i)differentiability for short) corresponds to the classic $H$-derivative introduced in [8], so this differentiability concept is a generalization of the Hukuhara derivative. Case (ii) of this definition corresponds to the second type Hukuhara derivative studied in $[\mathbf{9}, \mathbf{1 0}]$.

For an interval-valued function $F:\left[t_{0}, T\right] \rightarrow \mathbf{I}, F(t)=\left[F^{-}(t), F^{+}(t)\right]$, one defines the integral by the expression

$$
\int_{t_{0}}^{t} F(s) d s=\left[\int_{t_{0}}^{t} F^{-}(s) d s, \int_{t_{0}}^{t} F^{+}(s) d s\right] .
$$

By the Newton-Leibniz formula, one can write: if an interval-valued function $F$ is a second type Hukuhara differentiable on $\left[t_{0}, T\right]$, then $F\left(t_{0}\right)=F(t)+(-1) \int_{t_{0}}^{t} F^{\prime}(s) d s$.

Definition 2.3. A mapping $X:\left[t_{0}, T\right] \rightarrow \mathbf{I}$ is bounded, if there exists an element $M>0$ such that $H(X(t),\{0\}) \leq M$, for all $t \in\left[t_{0}, T\right]$.

Corollary 2.1 (see, e.g., [10]). Let $X:\left[t_{0}, T\right] \rightarrow \mathbf{I}$ be given. Denote $X(t)=\left[X^{-}(t), X^{+}(t)\right]$ for $t \in\left[t_{0}, T\right]$, where $X^{-}, X^{+}:\left[t_{0}, T\right] \rightarrow \mathbf{R}$. 
(i) If the mapping $X$ is (i)-differentiable (i.e., classical Hukuhara differentiable) at $t \in\left[t_{0}, T\right]$, then the real-valued functions $X^{-}, X^{+}$are differentiable at $t$ and $X^{\prime}(t)=\left[\left(X^{-}\right)^{\prime}(t),\left(X^{+}\right)^{\prime}(t)\right]$.

(ii) If the mapping $X$ is (ii)-differentiable at $t \in\left[t_{0}, T\right]$, then the real-valued functions $X^{-}, X^{+}$are differentiable at $t$ and $X^{\prime}(t)=$ $\left[\left(X^{+}\right)^{\prime}(t),\left(X^{-}\right)^{\prime}(t)\right]$.

3. Main results. Consider the following interval-valued Volterra integral equations

$$
X(t)=F(t)+\int_{t_{0}}^{t} K(t, s, X(s)) d s
$$

for all $t \in\left[t_{0}, T\right]$, where $F:\left[t_{0}, T\right] \rightarrow \mathbf{I}$ and $K: \mathcal{D} \times \mathbf{I} \rightarrow \mathbf{I}$, with $\mathcal{D}=\left\{(t, s) \in\left[t_{0}, T\right] \times\left[t_{0}, T\right]: t_{0} \leq s \leq t<T\right\}$.

Theorem 3.1. Let $N, M$ and $L$ be positive numbers. Assume that $F, K$ satisfy the following conditions:

(i) $F:\left[t_{0}, T\right] \rightarrow \mathbf{I}$ is continuous and satisfies $H(X(t), F(t)) \leq N$;

(ii) $K: \mathcal{D} \times \mathbf{I} \rightarrow \mathbf{I}$ is continuous and satisfies the Lipschitz condition with respect to $X$, i.e.,

$$
H(K(t, s, X), K(t, s, Y)) \leq L H(X, Y)
$$

for $(t, s, X),(t, s, Y) \in \mathcal{D} \times \mathbf{I}$.

In addition, if $H(K(t, s, X),\{0\}) \leq M$, then there is a unique solution of IVIE (3.1) on $\left[t_{0}, \mathbf{T}\right]$ where $\mathbf{T}=\min \left\{T-t_{0},(N / M)\right\}$.

Proof. In the proof of this theorem, we apply the method of successive approximations to construct a sequence of continuous functions $X_{n}$ : $\left[t_{0}, \mathbf{T}\right] \rightarrow \mathbf{I}$ as follows:

$$
X_{0}(t)=F(t), \quad X_{n}(t)=F(t)+\int_{t_{0}}^{t} K\left(t, s, X_{n-1}(s)\right) d s, \quad n \geq 1 .
$$

By the mathematical induction method, we can see that all $\left\{X_{n}\right\}_{n \geq 0}$ are continuous mappings on $\left[t_{0}, \mathbf{T}\right]$. Further $H\left(X_{n}, F(t)\right) \leq M\left(t-t_{0}\right) \leq$ $N$, for all $n \geq 1$. Then, for $t \in\left[t_{0}, \mathbf{T}\right]$, we have 
$H\left(X_{1}(t), X_{0}(t)\right)=H\left(\int_{t_{0}}^{t} K\left(t, s, X_{0}(s)\right) d s,\{0\}\right) \leq M\left(t-t_{0}\right) \leq N$.

By (ii) and (3.2), we find that

$$
\begin{aligned}
H\left(X_{n}(t),\right. & \left.X_{n-1}(t)\right) \\
& \leq H\left(\int_{t_{0}}^{t} K\left(t, s, X_{n-1}(s)\right) d s, \int_{t_{0}}^{t} K\left(t, s, X_{n-2}(s)\right) d s\right) \\
& \leq L \int_{t_{0}}^{t} H\left(X_{n-1}(s), X_{n-2}(s)\right) d s .
\end{aligned}
$$

In particular,

$$
\begin{aligned}
H\left(X_{2}(t), X_{1}(t)\right) & \leq L \int_{t_{0}}^{t} H\left(X_{1}(s), X_{0}(s)\right) d s \\
& \leq L \int_{t_{0}}^{t} M\left(s-t_{0}\right) d s=\frac{M L\left(t-t_{0}\right)^{2}}{2 !}, \quad t \in\left[t_{0}, \mathbf{T}\right] .
\end{aligned}
$$

Furthermore, if we assume that

$$
H\left(X_{n-1}(t), X_{n-2}(t)\right) \leq \frac{M}{L} \frac{\left[L\left(t-t_{0}\right)\right]^{n-1}}{(n-1) !}, \quad t \in\left[t_{0}, \mathbf{T}\right],
$$

then we have

$$
\begin{aligned}
H\left(X_{n}(t), X_{n-1}(t)\right) & \leq L \int_{t_{0}}^{t} \frac{M}{L} \frac{\left[L\left(s-t_{0}\right)\right]^{n-1}}{(n-1) !} d s \\
& =\frac{M}{L} \frac{\left[L\left(t-t_{0}\right)\right]^{n}}{n !}, \quad t \in\left[t_{0}, \mathbf{T}\right] .
\end{aligned}
$$

It follows by mathematical induction that (3.3) holds for any $n \geq$ 1. Consequently, the series $\sum_{n=1}^{\infty} H\left(X_{n}(t), X_{n-1}(t)\right)$ is uniformly convergent on $\left[t_{0}, \mathbf{T}\right]$, and hence the sequence $\left\{X_{n}\right\}_{n \geq 0}$ is uniformly convergent. It follows that there exists a continuous function $X$ : $\left[t_{0}, \mathbf{T}\right] \rightarrow \mathbf{I}$ such that $H\left(X_{n}(t), X(t)\right) \rightarrow 0$ as $n \rightarrow \infty$. Since

$$
H\left(K\left(t, s, X_{n}(s)\right), K\left(t, s, X_{n}(s)\right)\right) \leq L H\left(X_{n}(t), X(t)\right) \longrightarrow 0
$$


on $\left[t_{0}, \mathbf{T}\right]$ as $n \rightarrow \infty$ and since

$$
\begin{aligned}
H\left(\int_{t_{0}}^{t} K\left(t, s, X_{n}(s)\right) d s\right. & \left.\int_{t_{0}}^{t} K(t, s, X(s)) d s\right) \\
& \leq \int_{t_{0}}^{t} H\left(K\left(t, s, X_{n}(s), K(t, s, X(s))\right)\right) d s
\end{aligned}
$$

it follows that

$$
\lim _{n \rightarrow \infty} \int_{t_{0}}^{t} K\left(t, s, X_{n}(s)\right) d s=K(t, s, X(s)), \quad t \in\left[t_{0}, \mathbf{T}\right] .
$$

By (3.2), we obtain that

$$
X(t)=F(t)+\int_{t_{0}}^{t} K(t, s, X(s)) d s
$$

and so $X$ satisfies (3.1).

To prove the uniqueness, let $Y:\left[t_{0}, \mathbf{T}\right] \rightarrow \mathbf{I}$ be a second solution for (3.1), and

$$
Y(t)=F(t)+\int_{t_{0}}^{t} K(t, s, Y(s)) d s .
$$

Then, for every $t \in\left[t_{0}, \mathbf{T}\right]$, we get

$$
H(X(t), Y(t)) \leq \int_{t_{0}}^{t} L H(X(s), Y(s)) d s .
$$

By applying the Gronwall inequality, we get $H(X(t), Y(t)) \leq 0$, which completes the proof.

Remark 3.1. With the assumptions of Theorem 3.1, the existence and uniqueness of the solution for problem (3.1) can be obtained by using the contraction principle. Let $\mathbf{S}$ be the space of continuous functions from $\left[t_{0}, \mathbf{T}\right]$ into $(\mathbf{I}, H)$ with $H_{0}(Z, F) \leq N$, i.e., $\mathbf{S}=\{Z \mid$ $Z:\left[t_{0}, \mathbf{T}\right] \rightarrow \mathbf{I}$ continuous and $\left.H_{0}(Z, F) \leq N\right\}$ where $H_{0}(Z, F)=$ $\sup _{t \in\left[t_{0}, \mathbf{T}\right]} H(Z(t), F(t))$. We define an operator $\mathbf{A}: \mathbf{S} \rightarrow \mathbf{S}$ by

$$
\mathbf{A} Z(t)=F(t)+\int_{t_{0}}^{t} K(t, s, Z(s)) d s .
$$


Now we shall prove the operator $\mathbf{A}$ is a contraction mapping with the assumptions of Theorem 3.1.

Proof. Step 1. To prove that $\mathbf{A}: \mathbf{S} \rightarrow \mathbf{S}$, we have to prove that $\mathbf{A Z}$ is continuous and $\mathbf{A Z} \subset \mathbf{S}$. For each $t \in\left[t_{0}, \mathbf{T}\right]$, we get

$$
\begin{aligned}
= & H\left(F(t+h)+\int_{t_{0}}^{t+h} K(t+h, s, Z(s)) d s, F(t)+\int_{t_{0}}^{t} K(t, s, Z(s)) d s\right) \\
\leq & H(F(t+h), F(t))+\int_{t_{0}}^{t} H(K(t+h, s, Z(s)), K(t, s, Z(s))) d s \\
& +\int_{t}^{t+h} H(K(t+h, s, Z(s)),\{0\}) d s .
\end{aligned}
$$

By using assumptions (i) and (ii), we obtain that the right hand side of (3.4) tends to zero as $h \rightarrow 0$. So $\mathbf{A Z}$ is continuous. Let $Z \in \mathbf{S}$. We have

$$
\begin{aligned}
H_{0}(\mathbf{A Z}, F) & =\sup _{t \in\left[t_{0}, T\right]} H(\mathbf{A Z}(t), F(t)) \\
& =\sup _{t \in\left[t_{0}, T\right]} H\left(F(t)+\int_{t_{0}}^{t} K(t, s, Z(s)) d s, F(t)\right) \\
& \leq \sup _{t \in\left[t_{0}, T\right]} \int_{t_{0}}^{t} H(K(t, s, Z(s)),\{0\}) d s \\
& \leq M\left(t-t_{0}\right) \leq N .
\end{aligned}
$$

Therefore, $\mathbf{A Z} \in \mathbf{S}$.

Step 2 . We have to prove that $\mathbf{S}$ is a complete metric space. Indeed, let $\left\{Z_{n}\right\}$ be a sequence in $\mathbf{S}$ converging to $Z \in C\left(\left[t_{0}, \mathbf{T}\right], \mathbf{I}\right)$. We consider

$$
H_{0}(Z, F) \leq H_{0}\left(Z_{n}, Z\right)+H_{0}\left(Z_{n}, F\right) \leq \varepsilon+N
$$

for sufficiently large $n$ and all $\varepsilon>0$. So $Z \in \mathbf{S}$. Therefore, $\mathbf{S}$ is a complete metric space. 
Step 3. We have to prove that $\mathbf{A}$ is a contraction mapping. For $Z, W \in \mathbf{S}$, we get

$$
\begin{aligned}
H_{0}(\mathbf{A Z}, \mathbf{A W}) & =\sup _{t \in\left[t_{0}, T\right]} H\left(\int_{t_{0}}^{t} K(t, s, Z(s)) d s, \int_{t_{0}}^{t} K(t, s, W(s)) d s\right) \\
& =\sup _{t \in\left[t_{0}, T\right]} L \int_{t_{0}}^{t} H(Z(s), W(s)) d s \leq L \mathbf{T} H_{0}(Z, W) .
\end{aligned}
$$

Therefore, $\mathbf{A}: \mathbf{S} \rightarrow \mathbf{S}$ is a contraction only if $L \mathbf{T}<1$. Since $\mathbf{S}$ is a complete metric space and $\mathbf{A}$ is a contracting self-map on $\mathbf{S}$, it has a unique fixed point $Z \in \mathbf{S}$. This fixed point is the required unique solution to IVIE (3.1).

Theorem 3.2 (Existence and uniqueness). Assume that $F, K$ satisfy the following assumptions:

(i) $F:\left[t_{0}, T\right] \rightarrow \mathbf{I}$ is continuous and bounded;

(ii) $K: \mathcal{D} \times \mathbf{I} \rightarrow \mathbf{I}$ is continuous and satisfies the Lipschitz condition with respect to $X$, i.e.,

$$
H(K(t, s, X), K(t, s, Y)) \leq L H(X, Y)
$$

for $(t, s, X),(t, s, Y) \in \mathcal{D} \times \mathbf{I}, L>0$;

(iii) $K(t, s,\{0\})$ is bounded on $\left[t_{0}, T\right]$. If $L T<1$, then IVIE (3.1) has a unique solution $X$ on $\left[t_{0}, T\right]$.

Proof. One can obtain this result easily by using the methods of successive approximations and the contraction principle as in the proof of Theorem 3.1 and Remark 3.1, respectively.

Finally, we shall give some simple examples of an IVIE. We consider the following homogeneous Volterra integral equation

$$
X(t)=F(t)+\lambda \int_{0}^{t} k(t, s) X(s) d s, \quad t \in[0, T]
$$

where $F(t), X(t) \in \mathbf{I}$ and $k(t, s) \in \mathbf{R}$. 
Corollary 3.1. Suppose that $k:[0, T] \times[0, T] \rightarrow \mathbf{R}$ and $F:[0, T] \rightarrow \mathbf{I}$ are given continuous functions and $\lambda$ is an arbitrary parameter. If $|k(t, s)| \leq M$ for all $0 \leq t, s \leq T$, then equation (3.5) has a unique interval-valued solution.

Case 1. We assume that $\lambda \cdot k(t, s)>0$ and $F(t)=\left[F^{-}(t), F^{+}(t)\right]$, $X(t)=\left[X^{-}(t), X^{+}(t)\right]$. From equation (3.5), we obtain

$$
\left\{\begin{array}{l}
X^{-}(t)=F^{-}(t)+\int_{0}^{t} \lambda \cdot k(t, s) X^{-}(s) d s \\
X^{+}(t)=F^{+}(t)+\int_{0}^{t} \lambda \cdot k(t, s) X^{+}(s) d s \\
X^{-}(0)=F^{-}(0) \\
X^{+}(0)=F^{+}(0) .
\end{array}\right.
$$

Case 2. We suppose that $\lambda \cdot k(t, s)<0$. From equation (3.5), we obtain

$$
\begin{gathered}
\left\{\begin{array}{l}
X^{-}(t)=F^{-}(t)+\int_{0}^{t} \lambda \cdot k(t, s) X^{+}(s) d s \\
X^{+}(t)=F^{+}(t)+\int_{0}^{t} \lambda \cdot k(t, s) X^{-}(s) d s \\
X^{-}(0)=F^{-}(0) \\
X^{+}(0)=F^{+}(0)
\end{array}\right. \\
{\left[\begin{array}{l}
X^{-}(t) \\
X^{+}(t)
\end{array}\right]=\left[\begin{array}{l}
F^{-}(t) \\
F^{+}(t)
\end{array}\right]+\int_{0}^{t}\left[\begin{array}{cc}
0 & \lambda k(t, s) \\
\lambda \cdot k(t, s) & 0
\end{array}\right]\left[\begin{array}{l}
X^{-}(s) \\
X^{+}(s)
\end{array}\right] d s .}
\end{gathered}
$$

Example 3.3. Let us consider the interval-valued Volterra integral equation

$$
X(t)=[1,2] \cos (t)+\int_{0}^{t} X(s) d s .
$$

We transform problem (3.6) into the following ordinary integral equation system

$$
\left\{\begin{array}{l}
X^{-}(t)=\cos (t)+\int_{0}^{t} X^{-}(s) d s \\
X^{+}(t)=2 \cos (t)+\int_{0}^{t} X^{+}(s) d s \\
X^{-}(0)=1 \\
X^{+}(0)=2
\end{array}\right.
$$




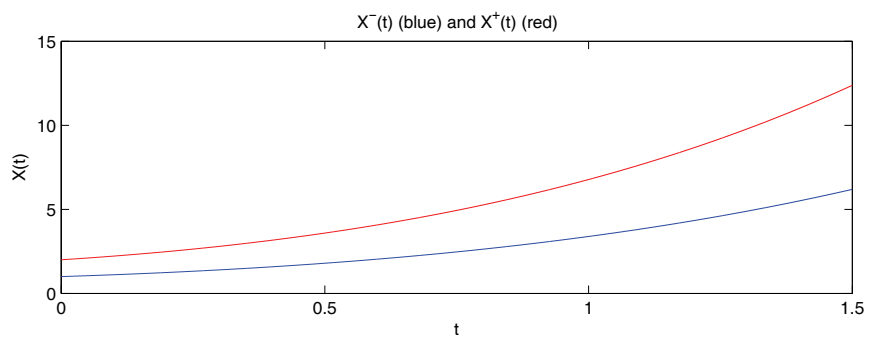

FIGURE 1. Solution of Example 3.3.

We obtain a unique solution to (3.6) defined on $[0, \pi / 2]$, and it is of the form

$$
X(t)=\left[\frac{-(\sin (t)+\cos (t))+3 e^{t}}{2},-(\sin (t)+\cos (t))+3 e^{t}\right] .
$$

This solution is illustrated in Figure 1.

Example 3.4. Let us consider the interval-valued Volterra integral equation

$$
X(t)=\left[t^{2}, 1+2 t^{2}\right]+\int_{0}^{t}-X(s) d s
$$

We transform problem (3.7) into the following ordinary integral equation system

$$
\left[\begin{array}{l}
X^{-}(t) \\
X^{+}(t)
\end{array}\right]=\left[\begin{array}{c}
t^{2} \\
1+2 t^{2}
\end{array}\right]+\int_{0}^{t}\left[\begin{array}{cc}
0 & -1 \\
-1 & 0
\end{array}\right]\left[\begin{array}{c}
X^{-}(s) \\
X^{+}(s)
\end{array}\right] d s
$$

We obtain a unique solution to $(3.7)$ defined on $[0,2]$, and it is of the form

$$
X(t)=\left[-e^{t}+2 e^{-t}+2 t-1, e^{t}+2 e^{-t}+t-2\right] .
$$

This solution is illustrated in Figure 2. 


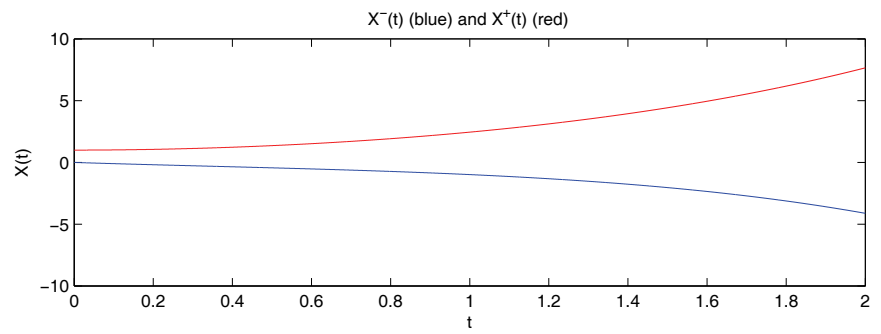

FIGURE 2. Solution of Example 3.4.

Acknowledgments. The authors would like to express their gratitude to the editor, Paul A. Martin, Prof. S. Sivasundaram and the anonymous referees for their helpful comments and suggestions, which have greatly improved the paper. The first named author would like to thank the University of Technical Education, Ho Chi Minh City, Vietnam.

\section{REFERENCES}

1. R.P. Agarwal, D. O'Regan and V. Lakshmikantham, Viability theory and fuzzy differential equations, Fuzzy Sets Syst. 151 (2005), 563-580.

2. - A stacking theorem approach for fuzzy differential equations, Nonlin. Anal.: Theory, Methods Appl. 55 (2003), 299-312.

3. Y. Chalco-Cano, A. Rufián-Lizana, H. Román-Flores and M.D. JiménezGamero, Calculus for interval-valued functions using generalized Hukuhara derivative and applications, Fuzzy Sets Syst. 219 (2013), 49-67.

4. F.S. De Blasi and F. Iervolino, Equazioni differenziali con soluzioni a valore compatto convesso, Boll. Unione Mat. Ital. 4 (1969), 491-501.

5. F.S. De Blasi, V. Lakshmikantham and T.G. Bhaskar, An existence theorem for set differential inclusions in a semilinear metric space, Contr. Cyber. 36 (2007), 571-582.

6. J. Vasundhara Devi, Generalized monotone iterative technique for set differential equations involving causal operators with memory, Inter. J. Adv. Eng. Sci. Appl. Math. 3 (2011), 74-83.

7. N.V. Hoa and N.D. Phu, On maximal and minimal solutions for set-valued differential equations with feedback control, J. Abstr. Appl. Anal. 2012, Article ID 816218, 11 pages, doi:10.1155/2012/816218.

8. V. Lakshmikantham, T. Gnana Bhaskar and J. Vasundhara Devi, Theory of set differential equations in metric spaces, Cambridge Scientific Publisher, Cambridge, 2006 . 
9. M.T. Malinowski, Interval differential equations with a second type Hukuhara derivative, Appl. Math. Lett. 24 (2011), 2118-2123.

10. - Interval Cauchy problem with a second type Hukuhara derivative, Inf. Sci. 213 (2012), 94-105.

11. - Random fuzzy differential equations under generalized Lipschitz condition, Nonlin. Anal.: Real World Appl. 13 (2012), 860-881.

12. - Existence theorems for solutions to random fuzzy differential equations, Nonlin. Anal.: Theory, Methods Appl. 73 (2010), 1515-1532.

13. - On random fuzzy differential equations, Fuzzy Sets Syst. 160 (2009), 3152-3165.

14. - On set differential equations in Banach spaces-A second type Hukuhara differentiability approach, Appl. Math. Comp. 219 (2012), 289-305.

15. - Second type Hukuhara differentiable solutions to the delay set-valued differential equations, Appl. Math. Comp. 218 (2012), 9427-9437.

16. S. Markov, Existence and uniqueness of solutions of the interval differential equation $X=f(t ; X)$, Compt. Rend. Acad. Bulg. Sci. 31 (1978), 1519-1522.

17. L.T. Quang, N.D. Phu, N.V. Hoa and H. Vu, On maximal and minimal solutions for set integro-differential equations with feedback control, Nonlin. Stud. 20 (2013), 39-56.

18. L. Stefanini and B. Bede, Generalized Hukuhara differentiability of intervalvalued functions and interval differential equations, Nonlin. Anal.: Theory, Meth. Appl. 71 (2009), 1311-1328.

Faculty of Foundation Sciences, University of Technical Education, Ho Chi Minh City, Vietnam

Email address: antv@hcmute.edu.vn

Faculty of Mathematics and Computer Science, University of Science, Ho Chi Minh City, Vietnam

Email address: ndphu_dhtn@yahoo.com.vn

Division of Computation Mathematics and Engineering (CME), Institute for Computational Science (InCOS), Ton Duc Thang University, VIETNAM

Email address: ngovanhoa_clt@yahoo.com 